\title{
Simulation in mental health interprofessional education
}

\author{
Cassandra (Sammy) lammarino*1 ${ }^{*}$ Jessie Johnson ${ }^{1}$, Monica Zolezzi ${ }^{2}$, Zohra Samnani Hasnani ${ }^{1}$ \\ ${ }^{1}$ Faculty of Nursing, University of Calgary in Qatar, Qatar \\ ${ }^{2}$ College of Pharmacy, Qatar University, Qatar
}

Received: June 3, 2020

DOI: $10.5430 /$ jnep.v10n10p46
Accepted: June 30, 2020

Online Published: July 1, 2020

\begin{abstract}
Purpose: Mental health care involves multiple professionals from diverse backgrounds providing interdependent and complex services. Accordingly, care needs to be planned skillfully in partnership with health and social care providers and mental health service users. Working from an interprofessional lens enables professionals to work collaboratively to affect care that is safe and improves health outcomes. Yet, in practice there is often a disconnect between mental health care professionals that hinders collaborative practice and impacts the quality of care. Furthermore, stigmatization of mental illness continues to pervade health care professionals' attitudes which serves to further compromise health outcomes. Interprofessional education (IPE) using simulation is proposed as an effective teaching and learning method to improve collaborative practice and decrease stigma amongst mental health care professionals in undergraduate education. Approach: Undergraduate nursing and pharmacy students from two universities participated in a one-day IPE event. During the event, students collaboratively interviewed standardized patients portraying mental health service users and developed an interprofessional plan of care. Faculty perspectives of the event were gathered to identify challenges and recommendations for ongoing implementation. Findings: Current literature and faculty facilitator feedback supports IPE using simulation as an effective teaching and learning strategy to develop therapeutic communication skills, address stigma amongst students prior to practice, clarify professional roles, and improve interprofessional collaboration. Faculty facilitator recommendations to improve the implementation of IPE with healthcare professionals during undergraduate education include early introduction of IPE, adequate preparation for students, realistic case scenarios, facilitator and standardized patient training, and funding to support events. Conclusion: The use of standardized patients in the context of interprofessional mental health education is a strategy with the potential to improve collaborative practice and address mental illness stigma amongst health care professionals. Further research with students is needed to evaluate the effectiveness of simulation in mental health IPE.
\end{abstract}

Key Words: Mental health, Interprofessional education, Simulation, Nursing education, Stigma

\section{INTRODUCTION}

Interprofessional collaboration, in which several professions work together to positively impact care, is necessary to improve the health and wellbeing of mental health service users. ${ }^{[1,2]}$ However, collaboration amongst mental health professionals can be a significant barrier to the provision of high-quality health care. Interprofessional education (IPE) is widely accepted as an effective strategy to improve interprofessional collaborative practice amongst health care providers1. IPE "occurs when two or more professions learn

\footnotetext{
*Correspondence: Cassandra (Sammy) Iammarino; Email: Cassandra.iammarino@ucalgary.edu.qa; Address: Faculty of Nursing, University of Calgary in Qatar, Qatar.
} 
about, from and with each other to enable effective collaboration and improve health outcomes". [3] Among undergraduate students, IPE has been shown to improve knowledge of professional roles and responsibilities, enhance attitudes towards other professions, facilitate communication, and prepare students for interprofessional practice. ${ }^{[4]}$ Secondly, the pervasive stigmatization of mental illness amongst healthcare providers and students is a major barrier to the provision of humanistic and inclusive mental health care. ${ }^{[5]}$ Stigma has devastating and wide-reaching effects for mental health service users and is often perpetuated in the clinical setting. The use of standardized patients as a context for IPE is proposed as a valuable learning methodology to encourage empathy and perspective taking amongst health professional students to address stigma prior to licensure as health care providers.

Simulation using standardized patients in interprofessional mental health education has the potential to address both poor collaborative practice and stigmatization of mental illness among healthcare students enrolled in mental health studies. This article describes a teaching innovation in which nursing and pharmacy students from two universities participated in a mental health IPE simulation using standardized patients. The event has been incorporated into the nursing and pharmacy undergraduate curricula at two universities in Qatar with the aim of improving role clarity, interprofessional collaboration, and provision of inclusive and appropriate care for mental health service users. Faculty facilitators discuss recommendations to improve the ongoing implementation of this teaching innovation and propose strategies for further evaluation.

\section{BACKGROUND}

Mental health service users often require complex mental health, physical health, and social care services. As such, interprofessional collaboration is particularly important in the provision of mental health services as these require effective working partnerships across varied disciplines. ${ }^{[6]}$ Collaborative mental health care has been shown to improve the quality of services and patient outcomes by facilitating knowledge transfer as well as improving access to mental health services and coordination of care across sectors. ${ }^{[4]}$ Clearly, mental health services users benefit when there is effective interprofessional collaboration. However, professionals working in mental health care may not always work well together. In fact, often there is a disconnect between professions that manifests in role stereotyping, lack of role clarity, as well as errors in the provision of patient care. ${ }^{[7,8]}$

Just as interprofessional collaboration is a vital component of mental health care, there is also a need to address the pervasive stigmatization of mental illness. Stigmatizing attitudes

Published by Sciedu Press and discriminatory behaviour can profoundly impact individuals' social, economic, and health status. In fact, mental health service users often identify stigma and discrimination as causing more harm than their illness. ${ }^{[9]}$ Stigma can negatively affect personal relationships, employment, and education. It can lead to discrimination and disadvantages in employment, income, and access to housing and healthcare, to name a few. Unfortunately, discriminatory attitudes and behaviors towards persons with mental illness are pervasive amongst healthcare providers and students. ${ }^{[5]}$ Evidently there is a need to address negative attitudes and behaviours and improve collaborative practice prior to professional practice early in undergraduate education.

IPE using standardized patients to realistically portray mental health service users is a valuable learning modality to improve collaborative practice necessary to increase knowledge and skills, address mental illness related stigma and ultimately improve outcomes for mental health service users. ${ }^{[8-10]}$ In recent years, the use of accurate and realistic simulated scenarios with trained standardized patients (SP) has gained popularity as an educational strategy for undergraduate students in health professions.

SP simulation involves the use of individuals trained to portray the roles of patients, family members, or care givers to allow students to improve important competencies (such as physical examination, history taking, interviewing, and counseling skills) before entry to practice. Simulation using standardized patients in which trained individuals portray mental health service users is a meaningful strategy to bridge knowledge and practice, and to encourage students to develop communication and collaborative practice skills in a low stakes environment. ${ }^{[11,12]}$ Moreover, the use of innovative teaching modalities such as simulation has been identified as a tool to address mental illness stigma by fostering understanding and increasing empathy towards people experiencing mental illness. ${ }^{[13]}$ The use of live, simulated, or video contact with mental health service users is identified as an effective teaching and learning method in IPE with the potential to address stigma amongst students. ${ }^{[9]}$ According to the contact method of stigma intervention, the use of standardized patients has the potential to improve perceptions of individuals with mental illness through interpersonal interaction. ${ }^{[14]}$

The use of standardized patients as a teaching modality in IPE can be used to address the dual challenges of collaborative practice and mental health stigmatization by improving interprofessional collaboration and enhancing empathy amongst students through the contact hypothesis. Maran$\operatorname{zan}^{[9]}$ argues that simulation as a learning method in IPE is 
an effective context for stigma reduction. Participation in IPE involves students assuming equal status with their peers and working collaboratively to achieve common goals. Stigma reduction interventions, through contact or knowledge-based approaches, align well with the goals and approach of IPE. While much is known about learners' perspectives and experience of IPE, there is a lack of faculty/facilitator perspectives on interprofessional education and few articles that outline a successful IPE event. ${ }^{[19]}$ This article provides faculty perspectives on a teaching innovation using standardized patient simulation as a teaching modality in a mental health interprofessional education event with nursing and pharmacy undergraduate students.

\section{APPROACH}

The IPE mental health activity has been offered once yearly since 2017 at the University of Calgary in Qatar (UCQ) Simulation Centre to second-year UCQ nursing students and fourth-year College of Pharmacy (CPH), Qatar University (QU) students (CPH-QU). A total of 262 nursing and pharmacy students have participated in this IPE activity since its inception (an average of 66 students per year). To ensure adequate preparation and experiential learning, the students must be currently enrolled or have completed their program's mental health theory and clinical rotations prior to the IPE event.

Faculty at UCQ and CPH-QU collaborated on the development and facilitation of the IPE activity. Faculty and clinical educators at the Simulation Center at UCQ developed the case scenarios. Careful consideration was taken in their development to ensure they were evidence-based, aligned with clinical practice, avoided stereotypes, and included comprehensive personal details for a fulsome portrayal of a mental health service user. CPH-QU faculty incorporated comprehensive medication histories and list of current medications to the scenarios. SPs were provided their scenarios in advance of the event and participated in a training session with a nursing faculty member to realistically portray symptoms of various mental disorders and discuss medications. Scenarios included diverse demographics including males and females from young adult to older adults with varied socioeconomic, ethnocultural, and medical histories. Scenarios included persons with obsessive compulsive disorder, major depressive disorder, bipolar disorder, generalized anxiety disorder, and substance use disorder. The level of acuity ranged from stable condition in an outpatient clinic to a higher acuity of suicidal ideation.

Prior to the simulation, faculty facilitators met to review the case scenarios, debriefing tool, and discuss learning objectives. Students reviewed relevant course content and were provided the opportunity to practice interviewing techniques. Students were then assigned to small groups consisting of two to three nursing and one to two pharmacy students. Prior to the interview, the students participated in an ice breaker activity to introduce themselves and their profession. The students briefly discussed their professional role and responsibilities in providing care in the mental health/psychiatric clinical setting to their group members. Following this, each small group engaged in a 15-minute collaborative interview with the SPs inside a simulation room with a two-way mirror. Interviews were observed outside of the room by the UCQ and CPH-QU faculty facilitators. The facilitator ensured interviews were completed on time and facilitated a post-interview 20-minute debriefing session with the students using the RUST model. ${ }^{[15]}$ SP's were also given the opportunity to provide brief feedback to students' about how they felt during each of the interviews. The faculty-facilitated debriefing session consisted of reflecting on the students' reaction to the interprofessional interaction with the SP including what went well, the challenges encountered during the interview, and the lessons learned from working with the other discipline and client. Lastly, the debriefing session was followed by collaborative development of a multidisciplinary plan of care which was summarized and illustrated as posters, each of which were presented by a representative of each group. The multidisciplinary care plans detailed the comprehensive client assessment, client-identified issues and, interventions and evaluation strategies from a nursing and pharmacy perspective. These integrated careplans included collaborative assessments, patient-centered issues, interventions, and evaluation within the nursing and pharmacist scope of practice.

The authors of this article have each participated in the planning and delivery of this event for at least two years. We discuss recommendations for ongoing planning and implementation of future mental health IPE activities.

\section{FINDings}

Faculty facilitators identified several key factors to the success of the mental health IPE event including realism, ability for learners to practice therapeutic communication in a lowstakes environment, and opportunity to learn more about the other profession. This feedback was evident through reflections written by students following participation in the event. Following the teaching innovation, faculty facilitators identified six recommendations for successful implementation of IPE amongst healthcare professional students in a mental health rotation: early introduction of IPE, adequate student preparation, realistic case scenarios, adequate training for standardized patients and facilitators, and funding. 


\subsection{Early introduction of IPE}

IPE must be introduced early and embedded in the curriculum. Through early introduction, it was postulated that learners will have greater exposure and experience learning with and about various professionals. IPE activities that link theory and practice should be intentionally planned and carried out from early in the program until graduation and include a variety of teaching and learning methods from case studies to simulations. This is particularly important in a mental health rotation where teamwork and collaboration are vital components of patient care.

\subsection{Adequate student preparation}

Students should be prepared prior to IPE event with relevant theory and experience. Prior to the event, learners should complete assigned readings and understand learning objectives to effectively make connections between theory and practice. Faculty advocated for learners to have some clinical exposure in mental health to ensure learning is applicable and relevant. Furthermore, faculty/facilitators identify that learners should have similar levels of experience to enhance collaboration and accurate understanding of roles and responsibilities. Avoiding disparities in skill levels amongst learners can attend to the power differentials that can occur across disciplines and role stereotyping.

\subsection{Realistic case scenarios}

Clinical-based case scenarios should emphasize a holistic view of the mental health service user and complex nature of mental health and illness. Attention must be paid to avoid reinforcing stereotypes or providing a reductive view of the mental health service user. Mental illness should be portrayed across the continuum of acuity and be inclusive of age, gender, socioeconomic status, and ethnocultural background. Case scenarios should go beyond the medical history to include a fulsome view of the individual. Furthermore, it would be beneficial to include mental health service users in the creation of realistic case scenarios. These strategies could challenge learners' assumptions and beliefs about mental health service users and challenge stigmatizing attitudes.

\subsection{Adequate training}

Standardized patients should receive training from a mental health professional on how to enact the case scenario condition in a realistic and non-stigmatizing manner. An in-person training session should be provided for an opportunity to practice, provide feedback, and discuss challenges. It is helpful for standardized patients to have access to educational videos, such as Symptom Media, to view accurate portrayals of symptoms of mental illness. It is important to avoid popular entertainment media portrayals as these are often rife

Published by Sciedu Press with stereotypes and misrepresentation.

Importantly, faculty facilitators should receive training prior to the IPE event to ensure skillful debriefing and encourage equal participation amongst healthcare students. First, faculty facilitators should become familiar with the purpose and competencies associated with the IPE event. Furthermore, they should receive training on the model used to facilitate reflection and debriefing of the simulation. It is suggested that skillful debriefing can enhance learner reflection on stigmatizing attitudes and discriminatory behaviors enacted in the simulation.

\subsection{Funding}

Institutional commitment to provide funding is necessary to ensure sustainability of IPE activities across the curriculum. Organization of an IPE activity is time consuming and involves participation from multiple institutions and faculty members. It requires intentional planning and resources to ensure success. Funding is necessary for administrative support, faculty time, student transportation, audio-visual and technical resources, adequate space, and evaluation. Crossinstitutional IPE committees should be established to enhance collaboration and effective planning.

\section{Discussion}

Faculty facilitators identified several factors necessary for a successful mental health IPE activity that align with the current literature. Importantly, faculty agreed that learners should be prepared prior to the simulation for application of theory to practice and meaningful learning to occur. In fact, learner preparation prior to IPE activities is tantamount to student success. ${ }^{[16]}$

Secondly, faculty facilitators agreed that providing students with the opportunity to engage in simulation in interprofessional education provides exposure to meaningful experiential learning while developing the skills necessary for interprofessional collaborative practice. Engaging students in realistic scenarios involving the use of SP's is a valuable tool in health professionals' education to improve mental health care. This particular activity enabled students the opportunity to interact with representative mental health service users in a safe learning environment, prepared them for further clinical practice where they would interview patients experiencing symptoms of acute mental health disorders, and enabled them to identify and develop collaborative plans of care. In this way, the realism of standardized patient simulations allows learners to increase their confidence through practice and consolidation of skills in a safe environment with timely feedback. ${ }^{[17]}$ 
Simulation in mental health IPE can provide a context to address mental illness stigma. Importantly, the IPE classroom can only address mental illness stigma if educators are well equipped and trained to incorporate effective learning methods and pedagogies to address the stigmatization of mental illness. ${ }^{[9]}$ Yet, it is of utmost importance that simulated scenarios draw from accurate and realistic clinical scenarios and standardized patients are highly trained to avoid reinforcing stereotypes or perpetuating stigmatizing beliefs. This is a vital task for educators in health care as stigma related to mental illness is not only prevalent amongst the public, it is also pervasive among healthcare students and professionals. ${ }^{[5]}$ Evidently, this should be a priority for educators to address through preparation on evidence-based pedagogies that impact change in attitudes and behaviors among health care professionals and students. ${ }^{[9]}$ Yet, the challenge for educators to successfully facilitate IPE is well documented in the literature. For many educators, teaching students during IPE is a new and difficult experience. ${ }^{[3]}$ For this reason, faculty facilitators require opportunities for professional development to learn more about IPE theoretical underpinnings and implications for practice. ${ }^{[18]}$

Lastly, institutional commitment to allocate funding is a barrier to the ongoing implementation of IPE embedded in curricula. The literature identifies a consistent barrier of resources including time provided to faculty to carry out the planning and delivery of IPE activities 19. Institutions must commit to human and material resources to engage faculty and change curriculum to include IPE. ${ }^{[19]}$

\section{Conclusion}

The use of standardized patients as a learning method in IPE has the potential to improve interprofessional collaborative practice and mental illness stigma reduction amongst healthcare students. This initiative can be implemented across contexts in the education of health care professionals working in mental health or other disciplines to address the disconnect between professions and pervasive stigmatization of mental illness. Interprofessional mental health simulation using SP's is an approach to learning that seeks to bridge the gap in understanding the different roles that professionals play when caring for mental health service users. It also affords the opportunity for students to learn with, from and about each other to affect change that is coordinated, collaborative and cohesive. Furthermore, it allows for important contact with simulated mental health service users to encourage a greater understanding and increased empathy to reduce stigma. In order to best implement IPE activities in undergraduate education, faculty feedback suggests it should be introduced early in curricula, students should receive adequate preparation, case scenarios should be realistic and reflect the experience of mental health service users, training should be provided for facilitators and standardized patients, and funding should be provided to embed IPE in curricula. While faculty voiced positive perspectives of IPE in mental health education, further research must be done to explore the effect of this teaching innovation on interprofessional collaboration and the impact on attitude change for students.

\section{CONFlicts OF INTEREST Disclosure}

The authors declare that there is no conflict of interest.

\section{REFERENCES}

[1] Reeves S, Perrier L, Goldman J, et al. Interprofessional education: effects on professional practice and health care outcomes. Cochrane Database of Systematic Reviews. 2013.

[2] Reeves S, Pelone F, Harrison R, et al. Interprofessional collaboration to improve professional practice and healthcare outcomes. Cochrane Database of Systematic Reviews. 2017.

[3] World Health Organization. Framework for Action on Interprofessional Education and Collaborative Practice. Geneva: WHO. 2010.

[4] Curran V, Heath O, Adey T, et al. An approach to integrating interprofessional education in collaborative mental health care. Academic Psychiatry. 2012; 36(2). PMid:22532196 https ://doi.org/10.1 176/appi.ap.10030045

[5] Thornicroft GT, Mehta N, Clement S, et al. Evidence for effective interventions to reduce mental-health-related stigma and discrimination. Lancet. 2016; 387: 1123-1132. https ://doi.org/10.1016/ S0140-6736 (15) 00298-6

[6] Pomare C, Long JC, Ellis LA, et al. Interprofessional collaboration in mental health settings: a social network analysis. Journal of Interprofessional Care. 2019; 33(5): 497-503. PMid:30411988 https://doi.org/10.1080/13561820.2018.1544550

[7] Barnes D, Carpenter J, Dickinson C. Interprofessional education for community mental health: Attitudes to community care and professional stereotypes. Social Work Education. 2000; 19(6): 565-583. https : //doi.org/10.1080/02615470020002308

[8] Barr H. Interprofessional education. In J.A Dent \& R.M Harden (4th Ed.), A Practical Guide for Medical Teachers (pp. 188-191). London: Churchill Livingstone; 2009.

[9] Maranzan KA. Interprofessional education in mental health: an opportunity to reduce mental illness stigma. Journal of Interprofessional Care. 2016; 30(3): 370-377. PMid:27152542 https: //doi.org/10.3109/13561820.2016.1146878

[10] Beebe LH, Roman M, Raynor H, et al. Transforming health care through interprofessional graduate education. Perspectives in Psychiatric Care. 2018; 54: 19-24.

[11] Baker C, Pulling C, McGraw R, et al. Simulation in interprofessional education for patient-centred collaborative care. Journal of 
Advanced Nursing. 2008; 64(4): 372-379. PMid:18764851 https: //doi.org/10.1111/j.1365-2648.2008.04798.x

[12] Attoe C, Kowalski C, Fernando A, et al. Integrating mental health simulation into routine health-care education. Lancet Psychiatry. 2016; 3(8): 702-703. https://doi.org/10.1016/S2215-0366(16) 3 0100-6

[13] Brown SA, Evans Y, Espenschade K. An Examination of Two Brief Stigma Reduction Strategies: Filmed Personal Contact and Hallucination Simulations. Community Mental Health Journal. 2010; 46: 494-499. PMid:20414723 https://doi.org/10.1007/s105 97-010-9309-1

[14] Corrigan PW, River LP, Lundin RK, et al. Three strategies for changing attributions about severe mental illness. Schizophrenia Bulletin. 2001; 27: 187-195. PMid:11354586 https://doi.org/10.1093/ oxfordjournals.schbul.a006865

[15] Karlsen KA. Stable Program. Adaptation of the RUS model. Original work from the Center for Medical Simulation (D.R.), Cambridge,
MA; 2013.

[16] Chou C, Ainsworth A, O’Brien B. An assessment strategy for interprofessional interactions of primary care practitioner trainees. Journal of Interprofessional Education and Practice. 2016; 2(2016): 1-3. https://doi.org/10.1016/j.xjep.2015.12.003

[17] Culpa-Bondal FA, Baker CD. Increasing students' self-confidence in patient interaction through standardized patient simulations, Sigma Repository, Conference Proceedings. 2016.

[18] Anderson ES, Cox D, Thorpe LN. Preparation of educators involved in interprofessional education. Journal of Interprofessional Care. 2009; 23(1): 81-94. PMid:19142786 https://doi.org/10.108 $0 / 13561820802565106$

[19] Loversidge J, Demb A. Faculty perceptions of key factors in interprofessional education, Journal of Interprofessional Care. 2015; 29(4): 298-304. PMid:25495176 https : //doi .org/10.3109/13 561820.2014 .991912 